\title{
Porcupine Inhibitor WNT974
}

National Cancer Institute

\section{Source}

National Cancer Institute. Porcupine Inhibitor WNT974. NCI Thesaurus. Code C116860.

An orally available inhibitor of porcupine (PORCN), with potential antineoplastic activity. Upon oral administration, WNT 974 binds to and inhibits PORCN in the endoplasmic reticulum (ER), which blocks post-translational acylation of Wnt lig ands and inhibits their secretion. This prevents the activation of Wnt ligands, interferes with Wnt-mediated signaling, and inhibits cell growth in Wnt-driven tumors. Porcupine, a membrane-bound O-acyltransferase (MBOAT), is required for the palmitoylation of Wnt ligands, and plays a key role in Wnt ligand secretion and activity. Wnt signaling is dysregulated in a variety of cancers. 